\title{
Image Matching for Aadhar Card Based on Textural and Geometrical Feature for Identification of Human
}

\author{
Anjali Swamy and Amit Yerpude \\ Department of Computer Science and Engineering, Rungta College of Engineering and Technology \\ Kohka road, Bhilai(C.G), India \\ anj.swamy1469@gmail.com; amit.yerpude@gmail.com
}

\begin{abstract}
Face appreciation from picture or film is a trendy topic in biometrics examine. Many civic places habitually have supervision cameras for film confine along with these cameras contain their large value for defense principle. It is broadly accredited that the features appreciation has played an imperative role in scrutiny system as it doesn't require the object's assistance. The genuine reward of features based discovery over added biometrics are rareness and approval. As creature expression is a energetic object having lofty degree of unevenness in its facade, that makes expression recognition a thorny trouble in mainframe idea. In this pasture, precision and rapidity of classification is a key matter.The target of this thesis is to appraise different expression discovery and appreciation method, offer whole clarification for reflection based visage recognition and detection with superior precision, enhanced reaction fee as an early stride for record inspection. resolution is projected based on perform tests on different look affluent database in requisites of subjects, facade, emotion, battle and radiance.
\end{abstract}

Keywords-SIFT features, Biometric security, Face recognition, Adhar card, Passport size photograph.

\section{Introduction}

During today's plexus humanity require to preserve the scrutiny of order or significant goods is fetching both gradually more imperative and tricky. periodically we listen regarding the crime of credit card con, processor breakings via hackers or defense crack in a corporation or direction structure.Biometric is automated process of recognize a self based on a physical or else behavioral characterstics.[2] Aadhar card as a verification of character which could be old to authenticate appearances of a person's own identity planned to allow clients that they be society of INDIA.Ballot chains is the vital dilemma of voting this paper is based on this method in which calculated results will be accurate. 


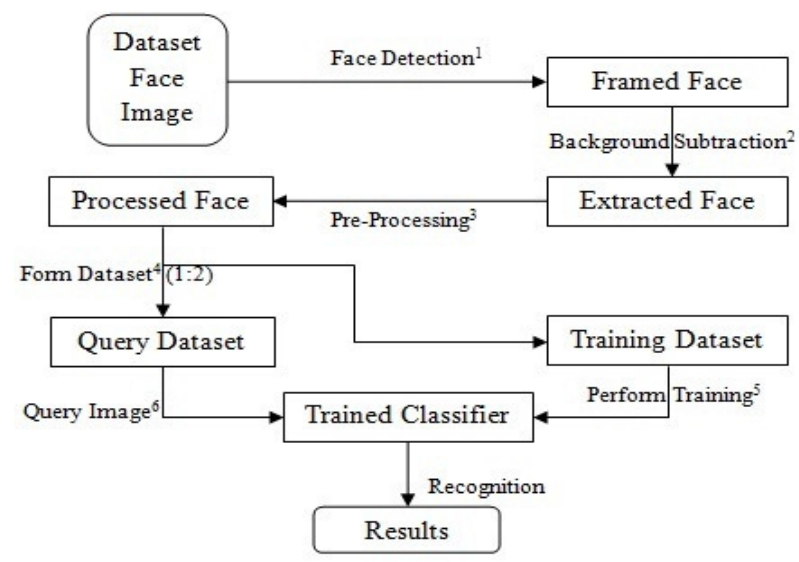

Figure 1. System Overview

Voting as an hour:- Finally ballet is an honour conducted between the citizens by the beginning father. By use their accurate to choose, people disclose their esteem for the the past of the nation. Today evoting machine(EVM) is used for ballet. This tries to look into e-voting from a diverse approach of the needed people sanction from a diverse slant in its place of concept such as OTP or smart card we endeavor to appear into the pro and con of biometric draw near.

\section{The Existing Ranking Methods}

Foregoing, The most popular procedure of keeping vote into the polling booth they used voter ID card as Authentication. These schemes desires the user to validate themselves by entering a address to validate that whether they are the citizens of that location or not.But when voter ID card are lost or not yet created at that time vote rigging will be done to make the vote for election which can be done by government.A biometric identification is one in which the user's face becomes the password/PIN biometric characterstics of an individual are unique and therefore can be used to authenticate a user's access to polling booth.

\section{User Query Intent and Storage Tags}

Sanctuary Experts says that Automatic Teller Machine (ATM) in potential will have biometric verification techniques toward validate identity of client through matter. In South America, near are company that include introduce fingerprint skill as a rooted piece of ATM system, where nation have previously ongoing using fingerprint in set of PIN or Password meant for broad detection with their ID cards.Gregg Rowley said- - Banks will move to smart cards and biometric will be next step after that[17] Electoral fraud can occur in advance of voting if the configuration of electorate is revised. The legitimacy of this type of manipulation varies across authority. Deliberate manipulation of election consequences is widely considered a violation of the principles of democracy.

\subsection{Vote Rigging:}

Vote rigging is an unauthorized interference with the process of an election acts of manipulation fraud affect vote counts to bring about an voting result. Whether by increasing the vote shared of the approved candidate distressing the vote share of the competitor candidate or both.[3]

\subsection{Corruption in democracy:}

As per [4] In a narrow election a small amount of crime may be enough to convert the result, Even if the outcome is not affected scam can still have a damaging effect if not a punished as it can diminish votersassurance in democracy. Even the awareness of scam can be damaging as it create people less 
apt to accept election result.Crooked election can margin to the disruption of democracy and the formulation of ratification of dictatorship.

\subsection{Misuse of proxy votes:}

As per [5] proxy voting is a particularly endangered to election scam, due to the aggregate of trust placed in the person who throw the vote.In several countries there have been assertion of evacuation home.citizens being asked to fill out Absentee voter form. When the form are signs and accumulated,they are then secretly recast as application for proxy votes, naming party opponent or their friends and relatives gives votes as proxies. But these people stranged to the voter then cast the vote for the party of their choice.This deception relies on elderly care home native typically being careless or suffering from insanity.

\section{A New Optimized Ranking Algorithm}

Biometric is the science that tries to fetch human biological facial appearance with an mechanized machine either to identify or authenticate. Biometric products eliminates the needs for password and PINS biometric system replace knowledge with entity features such as face recognition or immediacy detection. It makes it relaxed and fast to record features. The investigation of human data using the face recognition termed as the biometric.Initially the application of biometrics.[8]

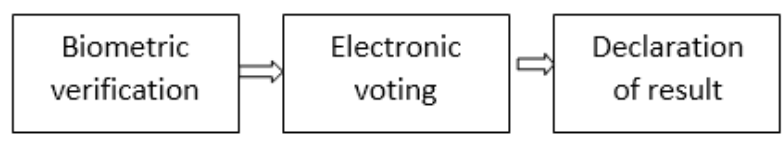

Figure 2: Biometric electronic voting architecture

\subsection{Biometric Registration of voters:}

This imply the method of capturing an appropriate(18 year of age or beyond with a sound mind a national and tenant in the country) Voter's personal information (Name,DOB, Home town, language,Address,Family,Passport,photography,etc.) with face recognition using recognition system and stored to the voters database be used for confirmation or certification on determination day.

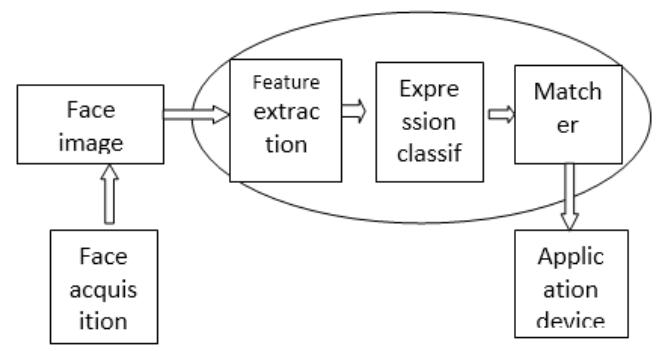

\subsection{Candidate Registration:}

Each supporting party or sovereign candidate after fleeting all the necessities of the electoral process obligatory for contesting in the selection would then be registered to the scheme after going during the ballot spot balloting apiece nominee individual information.

\subsection{Voter's Verification or Authentication:}

A face gratitude system operates moreover in verification approach or in recognition approach. 


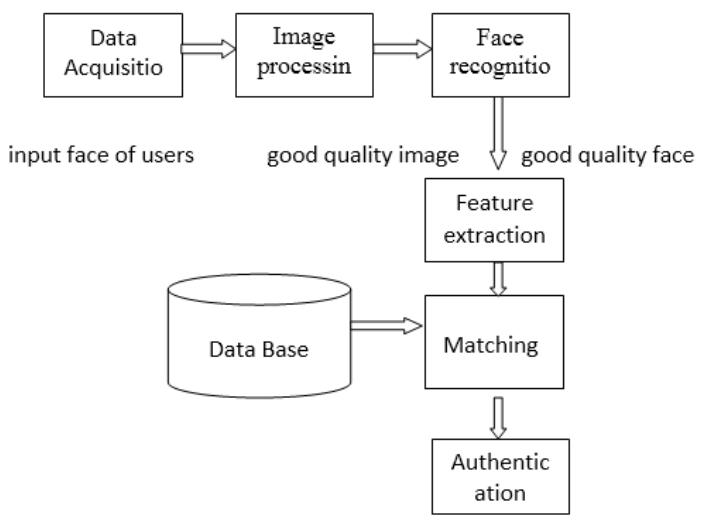

Figure 3: Architecture of face verification system

The first phase is the data acquisition in which a face image is obtained from an individual person. The next phase is the pre-processing phase in which the enter image is processed with some customary image processing algorithms for noise exclusion and smoothening. The pre-processed face icon is then improved via exclusively planned enrichment algorithms which exploit the episodic and directional life of ridges. The augmentation image is then used to dig out prominent features in the feature extraction stage.Finally the extracted textures are used for similar phase.[9]

\subsection{Face Verification:}

This paper introduces a prototype automatic identity authentication system that is capable of authenticating the identity of an individual using face images. The main algorithms are:

\subsubsection{Fiducial Point:}

A fiducial marker or fiducial is an object placed in the field of view of an imaging system which appears in the image produced, for use as a point of orientation or a measure. It may be either something placed into or on the imaging subject, or a spot or set of marks in the reticle of an optical instrument.In fiducial point length between eyes and nose will get calculated between similar images to detect the image whether it is matching or not.

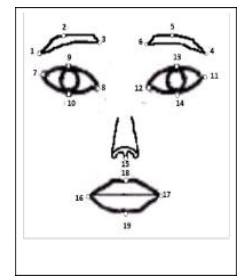

Figure 4: Architecture of fiducial points

Fiducial point is the detection between eyes nose and mouths. The performance of these tasks is usually to a large degree dependent on the accuracy of the facial point detector. It denote those detectors as facial component detectors. However, the cues for tasks like facial expression recognition or gaze detection lie in the more detailed positions of points within these facial components.Vectors $\mathrm{V} 1$ and V2 are nodes. The difference between the angles of the two vectors is ${ }^{\mathrm{a}}$ (alpha) and the ratio between the length of two vectors.

\subsubsection{Scale Invariant Feature Transform:}

SIFT is one of the most widely used technique of feature extraction. SIFT descriptor can transform image into scale invariant feature keypoints. The SIFT descriptor remain invariant under rotation ,scaling and variation in lightening condition. In original SIFT algorithm Euclidean distance is used for 
matching keypoints. This SIFT is used to smoothen the image and its points after getting the fiducial point. It will eliminate the unneccessary unstable points from the preceding algorithm. There are four major steps of SIFT algorithm:

- Scale space extrema detection: In this step candidate keypoints are detected.Image get convolved WithGaussianfiltersatdifferentscales

- and we take difference of successive Gaussian blurred image. DoG of image at different scales is $-D(x, y, \sigma)=L\left(x, y, k_{i} \sigma\right)-L(x, y, k j \sigma)$

Where $L(x, y, k \sigma)$ is convolution of original image.

$I(x, y)=$ Original image.

$\mathrm{G}(\mathrm{x}, \mathrm{y}, \mathrm{k} \sigma)=$ Gaussian blurred image

$L(x, y, k \sigma)=G(x, y, k \sigma) *{ }^{*}(x, y)$

After DoG obtained we have to find local maxima/minima of DoG image across scales for this-We have to compute each pixels in the DoG image to its eight neighbour at the same scale and nineth corrosponding neighbouring pixels in each of neighbouring scales. If the pixels is maximum/minimum among all compared pixels than the pixels is selected as a candidate keypoint.

- Keypoint Localization: After 1st step too many candidates points are generated in which some are stable and some are unstable.The main use of this keypoint is localization is to discard those points that have low contrast or poorly localized along an edge.

- Orientation Assignment: Gaussian smoothed image $L(x, y, \sigma)$ at the keypoint scale $\sigma$ is taken so that all computations are performed in a scale invariant manner.

For Example- A two parallel edges are also closed to each other and we use a strong gaussian filter these edge than merged

Let magnitude $=P(i, j)$ ( vertical deviation)

orientation $=\mathrm{Q}(\mathrm{I}, \mathrm{j})$ (horizontal deviation).

Wherever

$P(i, j)=[S(i+1)-S(i, j)+S(i+1, j+1)-S(i, j+1)] / 2]$

Where

$Q(i, j)=[S(i, j+1)-S(i, j)+S(i+1, j+1)-S(i+1, j)] / 2]$

Magnitude

$m(i, j)=\sqrt{ }(P(i, j)) *(P(i, j))+(Q(i, j)) *(Q(i, j))$

Orientation $\mathrm{K}(\mathrm{i}, \mathrm{j})=\arctan (\mathrm{Q}(\mathrm{i}, \mathrm{j}), \mathrm{P}(\mathrm{i}, \mathrm{j}))$

The Magnitude and direction are calculated for every pixel in a neighbouring region around the keypoint in the Gaussian blurred image L.

- Keypoint Descriptor: There are some steps of keypoint descriptor-

1. Find the blurred image of closest scale.

2. Sample the points around the keypoints.

3. Rotate the gradients and cordinated by the previously computer orientations. 
4. Seprate the region into sub region.

5. Create histogram for each subregions with 8 bins.

6. Weight the sample with $\mathrm{N}(\sigma)=1.5$ Region width

7. Trilinear interpolation(1-d factor) to place in histogram bins.

8. Actual implementations uses $4 * 4$ descriptors from $16 * 16$ which leads to a $4 * 4 * 8=128$ element vector.

\subsubsection{Sparsity face recognition:}

L1- minimization refers to finding the minimum I1- norm minimization solution to an undetermined linear system $b=A x . L 1$-norm minimization solution is the sparsest solution.It is used to compress the image and then store it into the database. To compress the image I1-norm minimization technique will be used.

\section{Experiment and Analysis}

Preprocessing is requisite since nearly everyone of the descriptions in the databases are sloping in some track. Therefore every metaphors are rotate in such a mode that a streak connecting eyes lies in a straight line. For textural system, a subspace be pick by relate features anthropometric calculate to stay away from the computational saddle by means of entire features. Worn to produce the subspace so as to contain needed details in the visage.Images of database tables are given in below Figure:

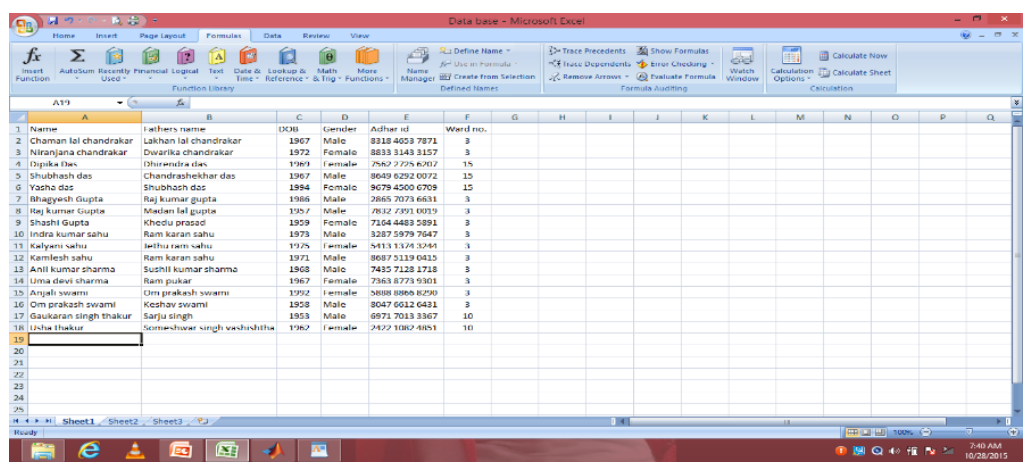

Figure 5: Database

The database contains the details of 17 peoples from which we had coleected the adhar card for their details and passport size photo for face detection with biometrics. The images of collected photos of persons are given below:

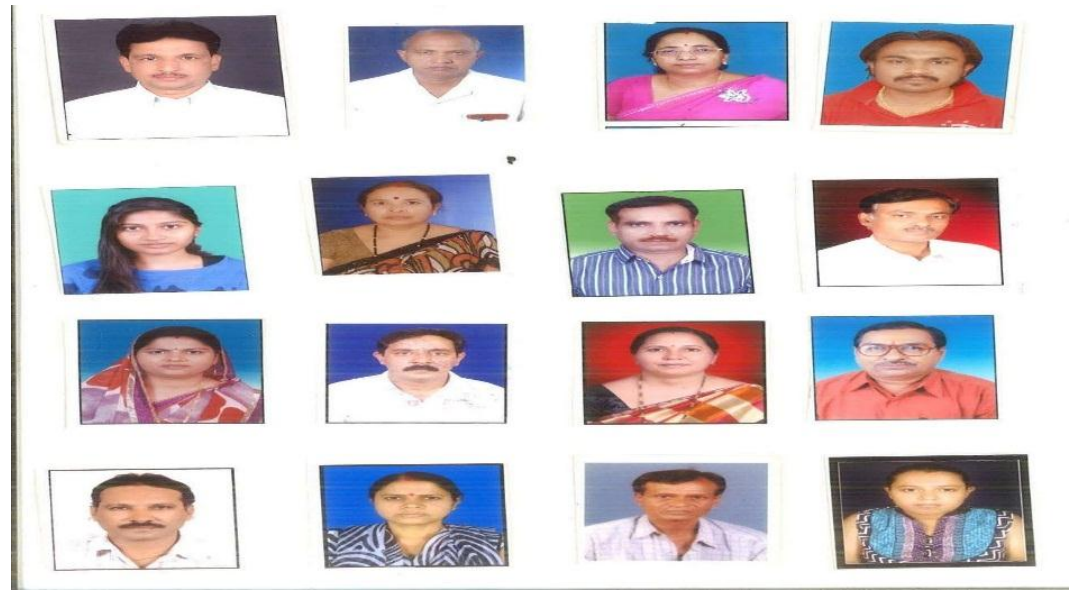

Figure 6: Passport images 
There are testing and training two features will be taken for biometric face recognition.The experimental result of testing features will be given below

\subsection{Testing features:}

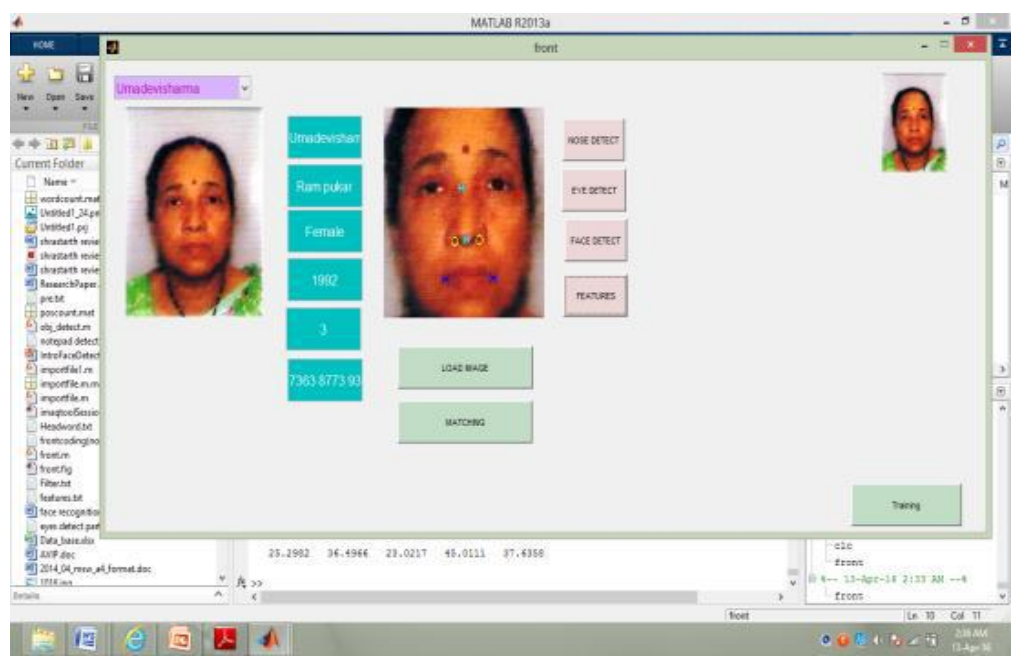

Figure 7: overview of matching

In testing features the length of eyes nose and lips will be calculated

Length of eyes 1 is $=25.2982$

Length of eyes 2 is $=36.4966$

Length of nose is $=23.0217$

Length of width of nose $=45.0111$

Length of Lips is $=37.6358$

Similarly value of all persons will be taken out and calculated.

\subsection{Training Features:}

Training features will be calculated from the images of adhar card faces and will be together calculated. 17 values calculated and each persong having 5 values i.e length of eyes 1 and eyes 2 , length of nose, width of nose, and length of lips.

1. $18.682,28.018,30.871,55.009,37.878$

2. $30.364,28.018,22.023,40.112,31.56$

3. $34.234,40.447,25,67.007,58.128$

4. $35.355,38.328,21.024,51.788,27.674$

5. $31.575,24.739,31.064,56.719,48.603$

6. $37.054,36.346,6.0828,56.009,30.586$

7. $38.013,38,38.21,58.078,40.321$

8. $37.216,39.051,35,62.129,40.672$

9. $37.054,40.792,11.402,63.388,42.695$ 
10. $23.087,30.265,27.074,53.151,41.904$

11. $34.713,36.77,40.497,46.011,24.923$

12. $35.228,38.079,5.099,57.219,21.516$

13. $34.132,39.623,49.659,62.37,34.587$

14. $29,41.304,30.017,44.181,44.196$

15. $30.414,37.336,22.023,47,36.147$

16. $42.012,40.012,30.806,59.414,35.228$

17. $39.013,31.145,25.02,52,35.732$

\subsection{Matching:}

Matching will be done by using KNN Algorithm in which min value be considered as a most matched imag. The images of database will get matched one by one with the passport images of the collected persons and then it will show the number of resultant image. The resultant image will be shown below:

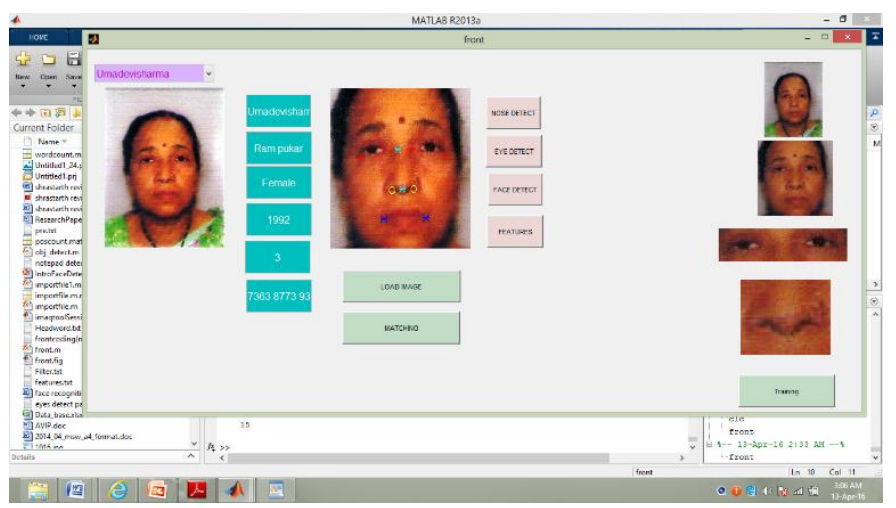

Figure 8: Matched image

6 Conclusions

The proposed framework can be analyzed in terms of feasibility and acceptance in the industry. Therefore trying to improve the performance of existing methods and introducing the new methods for face recognition based on today's software project requirement can be future work in this area.Our experiments indicate that better security than other methods like passwords, PIN Number.The benefits of multibiometrics may become even more evident in the case of a large database of users face recognition technology can be used worldwide to access buildings however it had been used in ATM's according to[7] but it can be used in voting machine which would help to reduce the vote rigging electoral fraud.

\section{REFERENCES}

[1] Lusekelo Kibona et al. Face Recognition as a Biometric Security for Secondary Password for ATM Users Ruaha catholic university(RUCU) Department of computer science Volume1,Issue2, 2015.

[2] I. Diebold "ATM fraud and security: White paper," Newyork , 2002 department of Computer Science and Engineering. 
[3] Anil Pandit and R.C Gangwar "Issues and challenges in electronic voting and direct recording electronic voting system". International Journal of Advanced Research in computer science and software engineering January 2015.

[4] Sarah Birch "Breifing paper on electoral corruption" at 05/11.At IDCR.

[5] Ines Levin, Gabe A. Cohn, Peter, C.Ordershoot and R. Michael Alvarez “Detecting voter fraud in an electronic voting context .An analysis of the Unlimited reelection vote in Venezuela, 23 june 2009.

[6] Riza Aditya Byoung Lee", Colin Boyd and Ed Dawson "Implementation Issues in secure e-voting schemes" at Joongbu University.

[7] S. Pravinthraja and Dr. K Umamaheshwari "Multimodal Biometrics for improving Automatic Teller Machine, December 2011.

[8] M.O Yinyeh, K.A. Gbolagade", Overview of Biometric electronic voting system in Ghana at july 2013.

[9] D.Ashok Kumar and T. Ummal Sariba Begum " A novel design of electronic voting system using fingerprint, International Journal of innovation technology and creative engineering, Vol1 pp.12-092011.

[10] Taigun Lee and Sung Kee Park and Mignon park, Department of electrical and electronic engineering "A new facial features and face detection method for Human Robot linteraction " IEEE april 2005.

[11] Paola Campaelli and Rafaella Lanzarotti " Localization of facial features and Fiducial points".

[12] Manju James and Shilpa Lizabeth George et al.,An Embedded Platform for Automtic Teller Machine using Finger- Vein Recognition System, International Journal of Industrial Electronics and Electrical Engineering Volume-2,Issue-2,Feb-2014 